\title{
Wheeze associated with prenatal tobacco smoke exposure: a prospective, longitudinal study
}

\author{
A L Lux, A J Henderson, S J Pocock, and the ALSPAC Study Team
}

\begin{abstract}
Aims-To determine whether maternal smoking during pregnancy is a risk factor for reported wheeze in early childhood that is independent of postnatal environmental tobacco smoke (ETS) exposure and other known risk factors.

Methods-A total of 8561 mothers and infants completed questions about smoking during pregnancy, ETS exposure, and the mother's recall of wheeze during early childhood.

Results-A total of 1869 (21.8\%) children had reported wheeze between 18 and 30 months of age, and $3496(40.8 \%)$ had reported wheeze in one or more of the three study periods (birth to 6 months, 6-18 months, 18-30 months). The risk of wheeze between 18 and 30 months of age was higher if the mother smoked during pregnancy. This relation did not show a dose-response effect and became less obvious after adjustment for the effects of other factors. Average daily duration of ETS exposure reported at 6 months of age showed a dose-response effect and conferred a similar risk of reported wheeze. Factors associated with early childhood wheeze had the following adjusted odds ratios: maternal history of asthma 2.03 (1.74 to 2.37$)$; preterm delivery 1.66 (1.30 to 2.13); male sex 1.42 (1.28 to 1.59$)$; rented accommodation 1.29 (1.11 to 1.51$)$; and each additional child in household 1.13 (1.04 to 1.24$)$.
\end{abstract}

Conclusions-Maternal smoking during pregnancy may be a risk factor for reported wheeze during early childhood that is independent of postnatal ETS exposure. For wheeze between 18 and 30 months of age, light smoking during the third trimester of pregnancy appears to confer the same risk as heavier smoking. (Arch Dis Child 2000;83:307-312)

Keywords: wheeze; maternal smoking; environmental tobacco smoke; longitudinal study

A systematic review of parental smoking and the prevalence of asthma or respiratory symptoms in children of school age ${ }^{1}$ has shown that there seems to be an effect of paternal smoking in households where the mother does not smoke. This observation suggests that some risk is conferred by postnatal exposure to tobacco smoke. However, the effect of maternal smoking appears to be stronger than that of paternal smoking. This may be because young children tend to be in contact with their mother longer than with their father. An alternative hypothesis is that prenatal exposure to tobacco smoke metabolites has an independent effect.

Some researchers have suggested that new prevalence studies are not justified, as there is strong evidence that cigarette smoke exposure is an important cause of wheeze. ${ }^{2}{ }^{3}$ They have suggested that new studies should compare critical periods of exposure to cigarette smoke, particularly in utero, and during infancy and early childhood.

We aimed to test the hypothesis that maternal smoking during pregnancy is an independent risk factor for wheeze during infancy and early childhood. We examined risk estimates before and after controlling for environmental tobacco smoke (ETS) exposure and other potential confounding factors. We also aimed to describe other important risk factors for reported childhood wheeze; and to estimate the relative importance of all significant risk factors by calculating adjusted population attributable fractions.

We obtained data from the Avon Longitudinal Study of Pregnancy and Childhood (ALSPAC). The protocol for this study has been published elsewhere, ${ }^{4}$ and information about the variables used can be obtained from the study website (http://www.ich.bris.ac.uk/ alspac.html). Women were enrolled if their expected date of delivery fell during or between April 1991 and December 1992.

\section{Subjects and methods}

The number of women with singleton infants who were eligible for enrolment in the study was 13870 . Of these, 12880 (92.9\%) completed the initial questionnaire, and 8561 $(61.7 \%)$ completed questionnaires that included information about the main exposures and outcomes. These variables were: smoking during the third trimester of pregnancy (during the two weeks preceding 32 weeks gestation); exposure of the infant to environmental tobacco smoke at 6 months of age; and childhood wheeze during three periods (from birth to 6 months; from 6 to 18 months; and from 18 to 30 months of age).

The outcome of primary interest was prevalence of wheeze during the third of these periods. We chose this period because we reasoned that earlier wheezing episodes were more likely to be caused by viral respiratory tract infections than to be a manifestation of asthma. Another outcome measure was the number of these periods in which wheeze was reported. We obtained information from questionnaires that were completed by the parents at the end of each of these periods. The question about 
wheeze asked whether there had been one or more episodes of wheeze or whistling on the chest since birth (or since the time of the previous questionnaire).

Maternal smoking at 32 weeks of pregnancy was self reported in one of four categories that gave information about the average number of cigarettes consumed per day during the preceding two weeks: none; 1 to $9 ; 10$ to $19 ; 20$ or more. We obtained information about ETS exposure from a questionnaire given to the parents when the child was 6 months old. This asked about the average number of hours exposed to ETS during weekdays and weekends. We summarised this with a variable which coded average reported daily ETS exposure in six categories: none; less than 1 hour; 1 to 2 hours; 2 to 4 hours; 4 hours or more; and "always".

We wished to look at factors that were potential confounders or effect modifiers of any relation we might find between smoking exposures and wheeze outcomes. We took information about physical, social, and environmental factors from a questionnaire administered in early pregnancy. Exploratory data analyses were used to see what effects these reported exposures might have, and some of the exposures were reduced to binary variables to ease interpretation of relative and attributable risks. For example, damp and mould in the home were both reported in four categories: very severe; fairly severe; not serious; and absent. Little information was lost by recoding these variables so that the first two categories were classified as "presence" and the last two categories were classified as "absence" of the risk factor. In a similar way, we recoded gestational age at delivery into two conventional groups: "term" if gestation was 37 weeks or greater, and "preterm" otherwise.

We defined five categories for duration of breast feeding: none; less than 1 month; 1 to 3 months; 3 to 6 months; and more than 6 months. The number of children in the household was that number reported before the infant being studied was born.

STATISTICAL METHODS

We used odds ratios (OR) as the main measures of relative risk, and we estimated adjusted odds ratios from logistic regression models. In order to measure the effects of potential risk factors on wheeze during more than one of the periods being studied, we used a cumulative odds ordinal logistic model. This is also described as a proportional odds model, or as an ordered polytomous logistic regression model. ${ }^{56}$ The model produces an odds ratio which summarises the relative risk of having wheeze in successively more of the periods being studied. It assumes that the odds ratio is uniform for each comparison of cumulative periods. In this case, the assumption is that the odds ratios for the following comparisons are the same: odds of wheeze in all three periods versus odds of wheeze in two or fewer periods; odds for two or three periods versus none or one period; and odds for one or more period versus none of the periods.
The main measures of attributable risk were adjusted population attributable fractions (PAFs). We adjusted the PAFs using estimates from the logistic regression model. All analyses were made with the Stata 5.0 statistical computing package. ${ }^{7}$

\section{Results}

At least one episode of wheeze was reported during the first six months after birth in 1764 $(20.6 \%)$ infants. The number reported to have wheeze between 6 and 18 months of age was $1971(23.0 \%)$, and the number with reported wheeze between 18 and 30 months of age was $1869(21.8 \%)$. Parents reported wheeze during one or more of the study periods in 3496 $(40.8 \%)$ children. The number of women who reported smoking during the third trimester of pregnancy was $1392(16.3 \%)$, and the number who reported some exposure of their infant to ETS at 6 months of age was 3016 (35.2\%).

There was a better completion of questionnaires by mothers with higher educational attainment. This trend had a high statistical significance, with completion ranging from $81.5 \%$ for mothers with university or polytechnic degrees to $56.6 \%$ for mothers whose highest educational attainment was a Certificate of Secondary Education (CSE). Table 1 shows the number and proportion of infants exposed to each risk factor.

Table 1 Distribution of risk factors

\begin{tabular}{|c|c|c|}
\hline & No. & $\%$ \\
\hline \multicolumn{3}{|l|}{ Maternal smoking during third trimester } \\
\hline None & 7169 & $(83.7)$ \\
\hline 1 to 9 cigarettes per day & 575 & $(6.7)$ \\
\hline 10 to 19 cigarettes per day & 618 & $(7.2)$ \\
\hline 20 or more cigarettes per day & 199 & $(2.3)$ \\
\hline \multicolumn{3}{|l|}{ Environmental tobacco smoke exposure } \\
\hline Never & 5545 & $(64.8)$ \\
\hline$<1$ hour per day & 1548 & $(18.1)$ \\
\hline 1 to 2 hours & 563 & $(6.6)$ \\
\hline 2 to 4 hours & 439 & $(5.1)$ \\
\hline 4 hours or more & 312 & $(3.6)$ \\
\hline "Always" & 154 & $(1.8)$ \\
\hline Maternal history of asthma & 933 & $(11.1)$ \\
\hline Preterm delivery & 356 & $(4.2)$ \\
\hline Male sex & 4447 & $(51.9)$ \\
\hline Living in rented accommodation & 1598 & $(19.0)$ \\
\hline \multicolumn{3}{|l|}{ Damp in home } \\
\hline None & 4402 & $(52.4)$ \\
\hline Not serious & 3597 & $(42.8)$ \\
\hline Fairly serious & 336 & $(4.0)$ \\
\hline Very serious & 61 & $(0.7)$ \\
\hline \multicolumn{3}{|l|}{ Mould in home } \\
\hline None & 6675 & $(80.0)$ \\
\hline Not serious & 1454 & (17.4) \\
\hline Fairly serious & 183 & $(4.0)$ \\
\hline Very serious & 33 & $(0.7)$ \\
\hline \multicolumn{3}{|l|}{ Number of children in home } \\
\hline None & 3779 & $(44.5)$ \\
\hline 1 & 3165 & $(37.2)$ \\
\hline 2 & 1184 & (13.9) \\
\hline 3 & 287 & $(3.4)$ \\
\hline$\geqslant 4$ & 84 & $(1.0)$ \\
\hline \multicolumn{3}{|l|}{ Number of previous pregnancies } \\
\hline None & 2878 & $(34.1)$ \\
\hline 1 & 2829 & (33.5) \\
\hline 2 & 1531 & (18.1) \\
\hline 3 & 742 & $(8.8)$ \\
\hline 4 & 274 & $(3.2)$ \\
\hline$\geqslant 5$ & 196 & $(2.3)$ \\
\hline Single marital status of mother & 1625 & $(19.2)$ \\
\hline \multicolumn{3}{|l|}{ Duration of breast feeding $(n=8358)$} \\
\hline None & 1835 & $(22.0)$ \\
\hline Up to 1 month & 1233 & $(14.8)$ \\
\hline Between 1 and 3 completed months & 1419 & $(17.0)$ \\
\hline Between 3 and 6 completed months & 1113 & (13.3) \\
\hline 6 or more completed months & 2758 & $(33.0)$ \\
\hline
\end{tabular}


WHEEZE OCCURRING BETWEEN 18 AND 30 MONTHS OF AGE

The proportion of children with reported wheeze in the period from 18 to 30 months of age was higher if the mother smoked $(27.7 \%$ versus $\left.20.7 \% ; \chi_{(1)}^{2}=33.1 ; \mathrm{p}<0.001\right)$ and if there had been ETS exposure $(24.8 \%$ versus $20.2 \% ; \chi_{(1)}^{2}=24.6 ; \mathrm{p}<0.001$; table 2$)$. There was a significant trend in the proportions of children with reported wheeze for increasing duration of ETS exposure $\left(\chi_{(\text {trend })}^{2}=8.32\right.$; $\mathrm{p}<0.01)$. There was no such trend for the number of cigarettes smoked during pregnancy $\left(\chi_{\text {(trend) }}^{2}=0.19 ; \mathrm{p}=0.67\right)$.

There was no reported exposure to either maternal smoking during pregnancy or ETS in $5190(60.6 \%)$ children. The number of children exposed to both was 1037 (12.1\%). The number exposed to ETS but not to maternal smoking during pregnancy was 1979 $(23.1 \%)$, and the number exposed to maternal smoking during pregnancy but not to ETS was $355(4.1 \%)$. Table 3 shows the effects of maternal smoking and ETS. We found no evi-

Table 2 Proportions with wheeze by average daily ETS and number of cigarettes smoked by mother during pregnancy

\begin{tabular}{|c|c|c|c|}
\hline & \multirow{2}{*}{$\begin{array}{l}\text { Number } \\
\text { without wheeze }\end{array}$} & \multicolumn{2}{|c|}{ Number with wheeze } \\
\hline & & No. & $\%$ \\
\hline \multicolumn{4}{|l|}{ Maternal smoking during pregnancy } \\
\hline None & 5685 & 1484 & $(20.7)$ \\
\hline 1 to 9 cigarettes per day & 421 & 154 & $(26.8)$ \\
\hline 10 to 19 cigarettes per day & 442 & 176 & $(28.5)$ \\
\hline 20 or more cigarettes per day & 144 & 55 & $(27.6)$ \\
\hline \multicolumn{4}{|l|}{ ETS exposure } \\
\hline Never & 4425 & 1120 & $(20.2)$ \\
\hline$<1$ hour per day & 1187 & 361 & (23.3) \\
\hline 1 to 2 hours & 428 & 135 & $(24.0)$ \\
\hline 2 to 4 hours & 322 & 117 & $(26.7)$ \\
\hline$>4$ hours & 227 & 85 & $(27.2)$ \\
\hline "Always" & 103 & 51 & (33.1) \\
\hline
\end{tabular}

Table 3 Odds ratios for wheeze due to maternal smoking during pregnancy and ETS exposure

\begin{tabular}{llll}
\hline & $\begin{array}{l}\text { Odds ratio } \\
\text { (males) }\end{array}$ & $\begin{array}{l}\text { Odds ratio } \\
\text { (females) }\end{array}$ & $\begin{array}{l}\text { Mantel-Haenszel } \\
\text { OR (95\% CI) }\end{array}$ \\
\hline $\begin{array}{l}\text { Maternal smoking during pregnancy } \\
\quad \text { First } 6 \text { months }\end{array}$ & 1.61 & 1.47 & $1.55(1.36$ to 1.77$)$ \\
$\quad$ to 18 months & 1.38 & 1.34 & $1.36(1.20$ to 1.55$)$ \\
$\quad 18$ to 30 months & 1.49 & 1.42 & $1.46(1.28$ to 1.67$)$ \\
ETS exposure & 1.31 & 1.34 & $1.32(1.19$ to 1.47$)$ \\
$\quad$ First 6 months & 1.32 & $1.15^{\star}$ & $1.24(1.12$ to 1.38$)$ \\
$\quad$ to 18 months & 1.30 & 1.33 & $1.31(1.18$ to 1.46$)$ \\
$\quad 18$ to 30 months & &
\end{tabular}

$\star 95 \%$ confidence interval includes unity.

Table 4 Odds ratios and adjusted odds ratios for risk factors for wheeze between 18 and 30 months of age

\begin{tabular}{|c|c|c|c|c|}
\hline & \multicolumn{2}{|c|}{ Odds ratio } & \multicolumn{2}{|c|}{ Adjusted odds ratio } \\
\hline & OR & $(95 \% C I)$ & $\overline{O R}$ & $(95 \% C I)$ \\
\hline Maternal smoking during pregnancy & 1.47 & (1.29 to 1.67$)$ & 1.19 & (1.02 to 1.39$)$ \\
\hline ETS & 1.31 & (1.18 to 1.45$)$ & 1.17 & $(1.03$ to 1.32$)$ \\
\hline Maternal history of asthma & 2.00 & (1.72 to 2.31$)$ & 2.03 & (1.74 to 2.37$)$ \\
\hline Preterm delivery & 1.59 & (1.26 to 2.01$)$ & 1.66 & (1.30 to 2.13$)$ \\
\hline Male sex & 1.42 & (1.28 to 1.57$)$ & 1.42 & (1.28 to 1.59 ) \\
\hline Rented accommodation & 1.57 & (1.38 to 1.77$)$ & 1.29 & (1.11 to 1.51$)$ \\
\hline Damp (fairly or very severe) & 1.46 & (1.16 to 1.82$)$ & 1.08 & (0.77 to 1.52$)$ \\
\hline Mould (fairly or very severe) & 1.36 & (1.00 to 1.84$)$ & 0.87 & (0.56 to 1.35$)$ \\
\hline Each additional child in household & 1.19 & (1.12 to 1.26$)$ & 1.13 & (1.04 to 1.24$)$ \\
\hline Number of previous pregnancies & 1.13 & (1.09 to 1.18$)$ & 1.01 & $(0.95$ to 1.08$)$ \\
\hline Single mother & 1.28 & (1.13 to 1.45$)$ & 1.07 & (0.92 to 1.24$)$ \\
\hline Breast feeding (any) & 0.88 & (0.77 to 0.99$)$ & 0.97 & $(0.85$ to 1.11$)$ \\
\hline
\end{tabular}

dence of effect modification of either smoking risk factor by sex.

The effect of ETS exposure after adjustment for the effect of maternal smoking during pregnancy by the Mantel-Haenszel method gave an odds ratio of 1.20 (1.07 to 1.34 ) and did not show significant effect modification $\left(\chi_{(1)}^{2}=2.44 ; \mathrm{p}=0.12\right)$. However, this interaction test has a low statistical power to detect a true difference in effects. The odds ratio for the effect of ETS exposure was 0.99 (0.75 to 1.29) for children of mothers who reported smoking during pregnancy and 1.25 (1.10 to 1.41$)$ for children whose mothers reported non-smoking during pregnancy.

Table 4 shows crude and adjusted odds ratios for smoking and other exposures. The strongest associations, after adjustment for the effects of all the other factors, were maternal history of asthma, preterm delivery, and male sex. The number of children in the household remained a significant risk factor after adjustment. Damp and mould were not significant after adjustment, but the effect of living in rented accommodation was. There was also an increased risk for each additional child in the household. Unmarried status of mother and number of previous pregnancies were not significant risk factors after adjustment.

A history of any breast feeding was associated with less reported wheeze $(21.3 \%$ versus $\left.23.7 \% ; \chi_{(1)}^{2}=4.58 ; \mathrm{p}=0.03\right)$, and there was a trend of smaller proportions with wheeze for longer duration of breast feeding $\left(\chi_{\text {(trend) }}^{2}=6.50 ; \mathrm{p}=0.01\right)$. However, the effect of breast feeding was not significant after adjusting for other significant risk factors.

WHEEZE DURING THE THREE STUDY PERIODS The cumulative odds ordered logistic model gave similar odds ratios for all of the comparisons described in the statistical methods section. The odds ratio tended to be highest for the comparison of odds of wheeze in all three periods against two or fewer periods, but there was no important heterogeneity. There is no accepted formal statistical test for heterogeneity of these odds ratios.

The summary odds ratios were similar to those obtained from the logistic regression model for wheeze between 18 and 30 months. However, this model suggests that maternal history of asthma (OR 2.18 (1.91 to 2.48)) and preterm delivery (OR 1.83 (1.48 to 2.25)) are marginally stronger risk factors for more persistent wheeze. The summary odds ratio for maternal smoking during pregnancy was 1.26 (1.11 to 1.42), and that for ETS exposure was 1.17 (1.06 to 1.29 ).

POPULATION ATTRIBUTABLE FRACTIONS

Table 5 shows the adjusted population attributable fractions (PAFs) for reported wheeze between 18 and 30 months of age. The model suggests that $33.6 \%$ of wheeze in the population is attributable to these risk factors. Attributable risks depend on both relative risk and the prevalence of risk factors. As ETS exposure is more common than maternal smoking during pregnancy, it has a higher attributable 
Table 5 Adjusted population attributable fractions (expressed as percentages) and 95\% confidence intervals for important risk factors from logistic regression model

\begin{tabular}{lrl}
\hline & \multicolumn{2}{c}{$\begin{array}{c}\text { Population attributable } \\
\text { fraction }\end{array}$} \\
\cline { 2 - 3 } & $\%$ & $C I$ \\
\hline Maternal history of asthma & 6.9 & $(5.2$ to 8.5$)$ \\
Preterm delivery & 1.8 & $(0.8$ to 2.7$)$ \\
Male sex & 13.9 & $(9.5$ to 18.0$)$ \\
Maternal smoking during pregnancy & 2.4 & $(0.2$ to 4.5$)$ \\
ETS exposure & 4.3 & $(0.8$ to 7.7$)$ \\
Rented accommodation & 4.0 & $(1.6$ to 6.4$)$ \\
Damp in home (fairly or very serious) & 0.3 & $(-1.0$ to 1.6$)$ \\
\hline
\end{tabular}

fraction $(4.3 \%$ versus $2.4 \%)$. Even though only $4.0 \%$ of the population were delivered preterm, this factor had a PAF of $1.8 \%$. The highest attributable fractions were those of male sex $(13.9 \%)$ and maternal history of asthma $(6.9 \%)$, but these are not amenable to public health interventions.

\section{Discussion}

The findings of this study suggest that maternal smoking during pregnancy has an effect on the prevalence of reported wheeze during early childhood, and that this effect is independent of the effects of environmental tobacco smoke exposure. We looked in detail at the period from 18 to 30 months of age, but we also used a cumulative odds ordered logistic model to show that these factors appear to be predictors of more frequent wheeze. The magnitude of the relative risk appears to be similar for both ETS and maternal smoking during pregnancy, and is modest. The adjusted odds ratios are 1.17 and 1.19 respectively, and the lower limits of the $95 \%$ confidence intervals are 1.03 and 1.02 .

PUBLIC HEALTH IMPORTANCE

The population attributable risk is higher for ETS than for maternal smoking during pregnancy because this is a more prevalent exposure. These PAFs refer to the binary risk factor "any versus no exposure". This study suggests that $6.7 \%$ of wheeze in early childhood could be prevented by modification of maternal smoking practices and ETS exposure. As wheeze was reported in $21.8 \%$ of children between the ages of 18 and 30 months, this attributable fraction corresponds with wheeze in $1.5 \%$ of all children in this age group. One study in the United States suggested that maternal smoking is responsible for $7.5 \%$ of all symptomatic cases of childhood asthma or wheezing lower respiratory tract illness. That corresponded with 380000 excess cases occurring during childhood in the United States. ${ }^{8}$

In our study, the poorer completion of questionnaires by mothers with lower educational attainments is not likely to have biased estimates of relative risk, but is likely to have led to an underestimation of the proportion of the population exposed to tobacco smoke. Thus, the PAF and the public health importance of these risk factors are likely to have been underestimated.
Maternal history of asthma, preterm delivery, and male sex were more important risk factors. However, they are less readily amenable to public health interventions.

\section{VALIDITY AND RELIABILITY}

We have considered whether the apparent effect of maternal smoking during pregnancy is caused by reporting bias or residual confounding, with the true effect being a result of ETS exposure. In our study, ETS exposure was based on reported duration of exposure and did not measure the number of cigarettes smoked. The number of cigarettes smoked is a poor surrogate measure for the concentration of tobacco smoke components in the environment. It is plausible and likely that duration is the more important risk modifier for ETS. We found that this measure showed a clear and statistically significant dose-response effect, and this suggests that the measure was valid and reliable.

We were interested to find that the odds ratio for ETS exposure was near 1 for children whose mothers did report smoking during pregnancy, but was 1.25 for children whose mothers reported not smoking during pregnancy. Even though this effect modification was not statistically significant, the finding is interesting. One interpretation is that mothers who smoked during pregnancy exposed their infants to ETS, but either did not report or did not recognise this exposure. They might, for example, have restricted smoking to other rooms in the house. A second interpretation is that some mothers smoked during pregnancy without reporting this, and the apparent effect of ETS is a result of the in utero exposure.

All data in the ALSPAC study are collected from contemporaneous questionnaires, which asked about current or recent exposures. The smoking exposure data were reported in categories rather than as continuous variables. This results in some loss of information, but it is not likely that exact numbers of cigarettes smoked or hours of ETS exposure would be reported with great accuracy. Even though ETS exposure had a clear dose-response effect, its inclusion in a logistic regression model as either a binary, categorical, or linear effect only minimally altered estimates of the effect of maternal smoking during pregnancy. However, we think that there is likely to be residual confounding by the ETS exposure effect.

\section{CAUSALITY}

Infants of women who smoke during pregnancy are more likely to have diminished lung function soon after birth ${ }^{9}$ and to wheeze during early infancy. ${ }^{1011}$ As the lungs grow, the propensity for wheeze that is associated with small airways diminishes, even though abnormalities of pulmonary function persist. ${ }^{12}$ Small airways might contribute to the propensity for wheeze before the age of 30 months, the upper age limit of the children in this study, and the wheeze might be provoked by ETS exposure. Passive inhalation of smoke might have a direct inflammatory effect on airway 
mucosa, or increase susceptibility to infection. ${ }^{13}$ However, as the child becomes older, abnormalities of lung growth and development caused by any in utero exposures are likely to become less important predictors of wheeze, and this might explain why our study did not find a dose-response effect for maternal smoking during pregnancy.

We found that a maternal history of asthma was strongly associated with wheeze between the ages of 18 and 30 months. There have been reports of increased serum IgE concentrations in infants whose mothers were smokers, ${ }^{14}$ but later studies have shown that this association is removed by adjusting for potential confounding factors. ${ }^{1516} \mathrm{~A}$ murine model suggests that exposure to ETS up regulates allergic responsiveness by means of Th2 cytokines. ${ }^{17}$ However, we are not aware of any studies that would show this effect in humans. A reasonable appraisal of current knowledge is that there is little evidence of a causal link between ETS exposure and the induction of inhalant allergy in children. ${ }^{18}$ There is, however, evidence that the effects of ETS exposure are different in atopic and non-atopic children. ${ }^{19}{ }^{20}$ It may be that ETS acts as an adjuvant to allergen induced inflammation of airways in atopic children but acts, in non-atopic children, by irritating airways directly or by influencing airway size.

Our data were based on the self reported smoking behaviour of women. The validity of these data might be questioned and our observations would have been more robust if we had prospectively measured serum or salivary concentrations in order to validate the reported smoking histories. However, one study that reported diminished lung function as a consequence of prenatal tobacco smoke exposure showed that reported smoking and serum cotinine concentrations were strongly correlated. ${ }^{9}$

Information about exposures was collected before information about outcomes was available, and any misclassification of exposures would be non-differential. Non-differential misclassification leads to underestimated risk estimates. In this study, ETS exposure is treated as a confounding factor for the association between in utero exposure and childhood wheeze, and we need to consider if its effects have been underestimated as a result of misclassification. We think that smoking exposure will have been over reported in few cases, and that maternal smoking during pregnancy is more likely to have been under reported than ETS exposure. Hence, the effects of in utero exposure are more likely to have been underestimated than those of ETS exposure.

We found that women reported smoking fewer cigarettes during pregnancy than before and after pregnancy. It is possible that the apparent lack of a dose-response effect of maternal smoking during pregnancy is a result of a tendency to under report the number of cigarettes smoked during pregnancy. However, it is quite plausible that many women were smoking fewer cigarettes while pregnant.
The absence of a dose-response effect for maternal smoking during pregnancy might make this exposure seem a weaker candidate for being a causal factor than ETS exposure. However, in utero exposure might mediate an effect by small concentrations of absorbed components or metabolites of tobacco smoke, and there may be a threshold effect at low levels of maternal smoking. From these data, we might infer that light smoking during the third trimester of pregnancy confers the same risk of wheeze between 18 and 30 months of age as heavier smoking.

It is difficult to design a study to distinguish the effects of in utero and environmental exposures to tobacco smoke, as very few infants exposed to maternal smoking during pregnancy are then reared in smoke free environments. We attempted to assess the relative effects of these exposures using data from a prospective, longitudinal study, and we have considered the potential effects of confounding factors, under reporting, and misclassification. However, it is likely that there remains residual confounding of the effect of prenatal smoking by that of ETS exposure.

Asthma is a complex, polygenic disease and its phenotypic expression is determined by interactions between genes and environment. The contribution of tobacco smoke to abnormalities of lung function and wheezing illnesses in children might appear subtle against a background of wide variations in normal growth and development of the lungs. ${ }^{21}$ We have shown small but statistically significant associations between wheezing in young children and both prenatal and postnatal tobacco smoke exposure. These effects were independent of other variables that were considered to be potential confounders or effect modifiers.

FUTURE STUDIES

Further follow up of this cohort of children will allow us to establish whether these exposures are important predictors of wheeze in later childhood. It will also allow us to determine whether early exposure to tobacco smoke influences objective measurements of atopy and pulmonary function.

The analysis of longitudinal data that have correlated exposure and response variables can be difficult, and valid analyses often require log linear models, marginal regression models, or generalised estimating equations. ${ }^{22}$ The models that we have used in this study are intuitively easier and may support the tentative inferences that we have made. However, more complex models would be needed to look at the effects of age related changes in the propensity to wheeze and in exposures to risk factors. We also think future studies will need to assess the validity and reliability of questionnaire data by making serial measurements of cotinine.

\section{CONCLUSIONS}

This study suggests that maternal smoking during pregnancy might be an independent risk factor for wheeze during infancy and early childhood. Our data and models suggest that the relative risks of smoking during pregnancy 
and ETS exposure are similar, though ETS exposure is associated with a higher attributable risk. The risk of wheeze caused by ETS increases with duration of exposure whereas, for wheeze between 18 and 30 months of age, light smoking during pregnancy appears to confer the same risk as heavier smoking.

We are extremely grateful to all the mothers who took part and to the midwives for their cooperation and help in recruit an to the midwives for their cooperation and help in recruitment SPAC study team comprises interviewers, computer technicians, laboratory technicians, clerical workers, research scientists, volunteers, and managers who continue to make the study possible. This study could not have been undertaken without the financial support of the Medical Research Council, the Wellcome Trust, the Department of Health, and the Department of the Environment. The collection of data fo this study was supported by the Asthma Research Council. The ALSPAC study is part of the WHO initiated European Longitudinal Study of Pregnancy \& Childhood. The initia analysis of these data formed part of an MSc thesis undertaken by Dr Lux at the London School of Hygiene \& Tropical Medicine. We should like to thank Dr Anthony R Brady, Imperial College School of Medicine, London, for providing the Stat program which calculates PAFs. We are also grateful to Dr Randall M Goldblum, Galveston, Texas and Dr Thomas R Martin, Boston, Massachusetts for comments on an earlier manuscript.

1 Cook DG, Strachan DP. Health effects of passive smoking. 3. Parental smoking and prevalence of respiratory symptoms and asthma in school age children. Thorax 1997;52:1081-94.

2 Stein RT, Holberg CJ, Sherrill D, et al. Influence of parental smoking on respiratory symptoms during the first decade of life. The Tucson Children's Respiratory Study. Am 7 Epidemiol 1999;149:1030-7.

3 Cook DG, Strachan DP. Health effects of passive smoking.

10. Summary of effects of parental smoking on children's 10. Summary of effects of parental smoking on children's respiratory health

4 Golding J. Children of the nineties. A longitudinal study of pregnancy and childhood based on the population of Avon (ALSPAC). West Engl Med $\mathcal{f}$ 1990;105:80-2

5 Greenland S. Introduction to regression models. In: Rothman KJ, Greenland S, eds. Modern epidemiology. Philadelphia, PA: Lippincott-Raven, 1998:359-99.

6 McCullagh P, Nelder JA. Generalised linear models. Boca Raton: Chapman \& Hall/CRC, 1989.

7 Stata statistical software (5.0). College Station, TX: Stata Corporation, 1996.
8 Stoddard JJ, Miller T. Impact of parental smoking on the prevalence of wheezing respiratory illness in children. Am $\mathcal{F}$ prevalence of wheezing resp

9 Stick SM, Burton PR, Gurrin L, Sly PD, LeSouef PN. Effects of maternal smoking during pregnancy and a family history of asthma on respiratory function in newborn infants. Lancet 1996;348:1060-4.

10 Martinez FD, Morgan WJ, Wright AL, Holberg CJ, Taussig LM. Diminished lung function as a predisposing factor for wheezing respiratory illness in infants. $N$ Engl F Med 1988; 319:1112-17

11 Dezateux C, Stocks J, Dundas I, Fletcher ME. Impaired airway function and wheezing in infancy: the influence of maternal smoking and a genetic predisposition to asthma. Am f Respir Crit Care Med 1999;159:403-10.

12 Martinez FD, Wright AL, Taussig LM, Holberg CJ, Halonen M, Morgan WJ. Asthma and wheezing in the first Halonen $M$, Morgan WJ. Asthma and wheezing in the first
six years of life. The Group Health Medical Associates. $N$ Engl f Med 1995;332:133-8.

3 Li JS, Peat JK, Xuan W, Berry G. Meta-analysis on the association between environmental tobacco smoke (ETS) exposure and the prevalence of lower respiratory tract infection in early childhood. Pediatr Pulmonol 1999;27:513.

14 Kjellman NI. Effect of parental smoking on IgE levels in children [letter]. Lancet 1981;1:993-4.

15 Ownby DR, Johnson CC, Peterson EL. Maternal smoking does not influence cord serum IgE or IgD concentrations. 7 Allergy Clin Immunol 1991;88:555-60.

16 Bjerke T, Hedegaard M, Henriksen TB, Nielsen BW, Schiotz PO. Several genetic and environmental factors influence cord blood IgE concentration. Pediatr Allergy Immunol 1994;5:88-94

17 Seymour BW, Pinkerton KE, Friebertshauser KE, Coffman RL, Gershwin LJ. Second-hand smoke is an adjuvant for T helper-2 responses in a murine model of allergy. 7 Immunol 1997;159:6169-75.

18 Strachan DP, Cook DG. Health effects of passive smoking. 5. Parental smoking and allergic sensitisation in children [published erratum appears in Thorax 1999;54:366]. Thorax 1998;53:117-23.

19 Chen Y, Rennie DC, Dosman JA. Influence of environmental tobacco smoke on asthma in nonallergic and allergic tal tobacco smoke on asthma in nonal
children. Epidemiology 1996;7:536-9.

20 Agabiti N, Mallone S, Forastiere F, et al. The impact of parental smoking on asthma and wheezing. SIDRIA Collaborative Group. Studi Italiani sui Disturbi Respirator nell'Infanzia e l'Ambiente. Epidemiology 1999;10:692-8.

21 Tager IB. Smoking and childhood asthma- where do we stand? [editorial; comment]. Am f Respir Crit Care Med 1998;158:349-51.

22 Fitzmaurice GM. Regression models for discrete longitudinal data. In: Everitt BS, Dunn G, eds. Statistical analysis of medical data: new developments. London: Arnold, 1998. 